\title{
Violence et corruption : cas de l'Algérie
}

\section{Djilali Hadjadj}

\section{(2) OpenEdition}

Journals

Édition électronique

URL : http://journals.openedition.org/apad/203

DOI : 10.4000/apad.203

ISSN : 1950-6929

Éditeur

LIT Verlag

Édition imprimée

Date de publication : 1 juin 2003

\section{Référence électronique}

Djilali Hadjadj, « Violence et corruption : cas de l'Algérie », Bulletin de l'APAD [En ligne], 25 | 2003, mis en ligne le 15 juin 2007, consulté le 07 septembre 2020. URL : http://journals.openedition.org/apad/203 ; DOI : https://doi.org/10.4000/apad.203

Ce document a été généré automatiquement le 7 septembre 2020.

Bulletin de l'APAD 


\title{
Violence et corruption : cas de l'Algérie
}

\author{
Djilali Hadjadj
}

Quelques repères historiques ou l'histoire d'un double monopole militaire et politique

1 L'histoire singulière de l'indépendance algérienne et de ses premiers pas redouble les héritages transmis. L'armée eut la part qu'on sait dans la libération de l'Algérie. Elle ne se résolut pas à la perte d'un rôle prééminent. En 1962, après cent trente-deux ans de colonisation française, une des plus destructrices qui aient été, et une guerre de libération particulièrement meurtrière, les Algériens aspiraient à la paix, à un peu de mieux être et à plus de liberté. Cependant les choses tournèrent autrement. Bien avant l'indépendance, des conflits étaient apparus entre le FLN (Front de Libération Nationale), le Gouvernement provisoire et l'Armée de libération, essentiellement autour de la question du pouvoir. Ces conflits reprirent de plus belle dès les premiers mois de l'indépendance, avec une tournure sanglante. Si l'armée fit le dos rond pendant les premières années de l'indépendance, c'est qu'elle n'était pas préparée, entre autres, à prendre seule le pouvoir. Avec sa politique désastreuse, Ben Bella, le premier Président, donna à l'armée l'occasion d'accéder au pouvoir par un coup d'Etat militaire en juin 1965, de ne plus le lâcher jusqu'en 1999 et de reléguer au second plan le FLN. Les structures d'autorité de l'armée se transmirent au pouvoir lui-même, écartant la possibilité d'un développement démocratique pacifique. L'armée devint garante de toute légitimité, même s'il fallait de temps en temps en réaffirmer l'essence révolutionnaire.

Depuis plus de quarante ans, l'armée a détenu les pouvoirs essentiels et chaque crise ramène les mêmes noms aux postes de commande. Dans ces conditions, aucun élargissement des structures politiques n'est vraiment possible, Le monopole de la classe politique issue de l'armée a concentré les pouvoirs économiques, permis le développement en serre de la corruption et s'inscrit ainsi comme une nécessité pour les clans au pouvoir. Les liens qu'elle tisse occupent une place démesurée dans les décisions sociales, économiques et politiques. Toute idée de transformation est soumise, pour ces hommes, à la volonté de perdurer. 
31988 aura été l'année décisive, l'année d'une grande crise. La pénurie de produits alimentaires de base s'était installée du fait de la baisse sensible des importations consécutive à la chute de plus de moitié des revenus pétroliers. La révolte grondait dans les milieux ouvriers et populaires, d'autant plus que le pouvoir avait mis les syndicats au pas. Au sein du pouvoir, les luttes de clans s'exacerbaient. A partir de 1986, des manifestations populaires (dans le prolongement de la première crise pétrolière où les prix s'effondrèrent), dues à un profond mécontentement social eurent lieu dans plusieurs villes d'Algérie. Les premiers groupes islamiques armés qui venaient de faire leur apparition, furent rapidement anéantis par le pouvoir militaire. Du 5 au 10 octobre 1988, d'importantes manifestations éclatèrent dans les grandes villes algériennes. En particulier à Alger où les manifestants saccagèrent les édifices publics, se lancèrent dans le pillage des magasins d'Etat et clamèrent des slogans conspuant le Président et ses sbires en les accusant de corruption. La guerre des chefs était projetée dans la rue. L'armée intervint pour "rétablir l'ordre". La répression fut terrible : plus de cinq cents morts et des milliers de personnes torturées. L'introduction du pluralisme politique à partir de la constitution de 1989 , qui a pourtant mis fin au système du parti unique, n'a rien changé à ces pratiques.

4 En raison du très lourd endettement extérieur de l'Algérie et des faibles performances de l'économie, la situation sociale continua à se dégrader très rapidement. Cet état de fait ne cessa de profiter aux islamistes qui, exploitant la déroute du FLN, le faible ancrage des forces démocratiques dans la société, la marginalisation de masse et l'exclusion, dénonçaient sans trêve la corruption du pouvoir. Les barons de la grande corruption, installés au sein des institutions de l'Etat, étaient prêts à pactiser avec les islamistes (même radicaux) non seulement pour protéger leurs réseaux et leur butin, et éviter d'avoir à rendre des comptes, mais aussi pour se trouver encore aux premiers postes lors de la libéralisation de l'économie, source de nouveaux dividendes criminels. Après avoir tiré les leçons des victoires électorales écrasantes du Front Islamique du Salut en 1990 et 1991 (tant aux municipales qu'aux législatives), le pouvoir militaire annula les élections remportées par les islamistes, poussa à la démission le président Chadli au tout début de l'année 1992 (lui-même issu de l'armée et en place depuis 1979) et instaura une période de transition. Ces évènements marquèrent le début de l'action armée des islamistes, rejoints par des milliers de jeunes victimes de l'exclusion sociale, dont nombre d'entre eux avaient été profondément marqués par la violente répression d'octobre 1988. Le pouvoir militaire fit appel, en janvier 1992, à un ancien leader historique du FLN, Mohamed Boudiaf, qui s'était signalé dès l'indépendance par une autonomie certaine à l'égard du pouvoir et était favorable à plus de liberté d'expression et d'ouverture politique. Boudiaf, placé à la tête de l'Etat, s'aperçut vite de l'étendue de la corruption dans laquelle étaient impliqués de hauts dignitaires du régime. Il multiplia les interventions dans lesquelles il dénonçait sans faiblesse "la mafia politico-financière". Trois mois après son arrivée, il fit part de sa détermination à lutter contre la corruption. Le 29 juin 1992, lors d'une rencontre avec des jeunes et des associations, Mohamed Boudiaf fut mitraillé. Sa mort avait été filmée et ses images partiellement retransmises à des millions d'Algériens atterrés. Le tout-sécuritaire mis en pratique par le pouvoir à l'encontre des militants islamistes et des groupes armés, la libéralisation sauvage de l'économie, les atteintes répétées aux droits de l'homme et la limitation des libertés démocratiques marquèrent cette longue période dite de transition, dans l'attente de nouvelles élections. 
5 Lors de l'été 1993, vacances scolaires aidant, le départ massif des élites s'accentua, vidant le pays de ses cadres et de ses intellectuels. La terreur s'installa dans la société civile et les démocrates de tous bords furent particulièrement pris pour cible. Le contexte parut propice aux barons de la corruption, excédés par les caisses vides de l'Etat et les conséquences catastrophiques que cela avait sur la "santé" de leurs affaires. Ceci les amena à pousser les décideurs politiques à précipiter un rapprochement avec le FMI et rapidement, un accord s'appuyant sur un reprofilage multilatéral de la dette, fut signé. Les chefs des réseaux de la corruption se frottaient les mains : le remboursement de la dette allait connaître un répit durable et de l'argent frais serait de nouveau disponible par milliards de dollars. En 1993, l'Algérie devait une somme de près de 30 milliards de dollars. Puis, grâce au rééchelonnement de la dette, la facture des importations put doubler d'une année sur l'autre pour atteindre 9 milliards de dollars. Comme la violence et l'insécurité s'aggravaient de jour en jour, bloquant les investissements productifs nationaux et étrangers, les conditions étaient réunies pour relancer la politique mafieuse de l'import-import". Cette expression couramment utilisée, fait référence à l'économie de l'Algérie qui n'exporte pratiquement rien d'autre que le pétrole et le gaz et importe tout ce dont elle a besoin. Après avoir dissous le Front Islamique du Sai ut et s'être assurée que les autres courants islamistes n'avaient aucune chance de l'emporter, l'armée a remis en marche le processus électoral dès 1995 pour les présidentielles. En 1997 les législatives connurent une fraude à grande échelle au profit du parti du général président Zéroual, coopté par ses pairs de l'armée dès 1994 à la tête de l'Etat. Ainsi le pouvoir, après avoir abusé de la "légitimité révolutionnaire" pendant trente-cinq ans, put-il se prévaloir d'une légitimité démocratique.

6 Le monopole politique s'habillait de pluralisme pour la continuité. Le départ précipité de Zéroual et le choix par les militaires de Bouteflika pour le remplacer dès le 15 avril 1999 (après un semblant d'élection) s'inscrivent dans la même logique. Cinq années plus tard, les militaires lui apportèrent de nouveau leur soutien pour un second mandat.

7 Aujourd'hui, grâce à la très forte augmentation des recettes pétrolières, le système du parti unique se refait une santé. Les dissensions entre Bouteflika et l'armée ont fait l'objet de longs commentaires dans la presse écrite algérienne (relayée par la presse étrangère) qui a renoncé à maintenir un point de vue autonome en se rangeant en majorité du côté des généraux (qui n'en demandaient pas tant) et en exigeant le départ de Bouteflika.

8 Monopole militaire, monopole politique, monopole économique, un seul monolithe s'est créé : celui du pouvoir hérité de la guerre de libération. La période de l'idéal soutenu par quelques hommes intègres et exprimé dans un socialisme de casernes est passée. Les sirènes du libéralisme ont tout emporté : absence de démocratie, mirage du pétrole et de la privatisation, voilà les ferments de l'explosion de la corruption alimentée par les flux extérieurs. Le pouvoir est à peine entrouvert aux seuls courants conservateurs et fondamentalistes. Les intégristes islamistes et le pouvoir en place font cause commune. Le terrorisme et la violence du pouvoir font 200000 morts et 20000 disparus. Le 7 octobre 2003, l'Algérie apparaissait en 88ème place dans le classement de Transparency International sur la perception de la corruption, avec un très faible score signifiant un haut niveau de corruption. En 2004, ce monopole politique avec toutes ses dérives anti-démocratiques et liberticides, demeure omniprésent. 
La malédiction du pétrole

Nombre d'observateurs et de spécialistes de l'Algérie s'interrogent à juste titre: comment ce pays, qui possède un potentiel humain magnifique, un territoire gigantesque, une histoire millénaire et de fantastiques ressources naturelles, peut-il plus de quarante années après son indépendance, compter près de la moitié de la population au-dessous du seuil de pauvreté et parallèlement, une caste de nouveaux riches issus des cercles du pouvoir?

Pour comprendre l'avènement et l'extension de la corruption en Algérie, il est essentiel de revenir sur l'exploitation des hydrocarbures d'économie. En effet, l'"or noir" nourrit depuis des décennies une véritable culture de la rente. Cette forme de pensée mortifère place en toutes circonstances le calcul égoïste et borné de l'argent facile à répartir, au-dessus de la préoccupation de l'avenir d'un peuple. N'est-ce pas là, une des sources principales de tous les malheurs qui se sont abattus sur ce pays depuis l'indépendance? Les retombées financières de l'économie "mono-exportatrice" de pétrole, après la nationalisation des hydrocarbures en 1971 et le premier choc pétrolier de 1973, permirent de lancer, au détriment de l'agriculture, un vaste programme d'industrialisation. La corruption y trouva un aliment surabondant. Ce que les pays occidentaux ont "perdu" suite au choc pétrolier, ils l'ont récupéré très largement par la conclusion de marchés faramineux, notamment dans le secteur de l'armement qui donnèrent lieu à d'abondantes commissions alimentant les dictatures et les réseaux de la corruption. De 1973 à 1984, l'argent du pétrole rentrait à ne plus savoir qu'en faire. Douce période pour les versements occultes, comme l'explique Pierre Péan dans son livre, L'argent noir (1988) : "dans l'arsenal mis en place pour récupérer la part de notre richesse nationale amputée par l'OPEP, la corruption tient alors une place déterminante (...), des dizaines de milliards de francs ont ainsi été dérivés vers des comptes en banque secrets de présidents, princes, ministres et hauts fonctionnaires des pays pauvres". A partir de 1986, les crises dues aux chutes durables des prix du pétrole mirent à nu les conséquences désastreuses de ce type d'économie mono-exportatrice. En décembre 1998, les prix du pétrole étaient au plus bas, au grand dam du pouvoir qui voyait déjà ses "affaires" décliner. Cette situation faisait augurer un troisième rééchelonnement de la dette qui aurait mis définitivement l'Algérie en faillite. Or le prix du pétrole a connu une forte augmentation à partir du second semestre de 1999 et a dépassé des mois durant, 30 dollars le baril. Cette hausse est toujours présente en 2004, pour la cinquième année successive. Les bénéficiaires de l'"import-import" retrouvèrent espoir. Pour François Gèze (2002), "cette corruption est le moteur fondamental de la 'sale guerre' que les 'décideurs' militaires mènent contre leurs compatriotes: sa fonction première est de briser et d'éradiquer tous les germes qui pourraient entraîner le peuple dans une révolution risquant de mettre fin à leurs privilèges et de tarir définitivement les sources de leur fortune".

Insécurité, violence, terrorisme et corruption

11 En 1996, sous prétexte d'état d'urgence (en vigueur depuis 1992 et sans interruption à ce jour), le ministère algérien de l'intérieur ordonnait à la presse nationale, notamment de statut privé, de s'en tenir aux communiqués officiels en matière d'information sécuritaire. Ce qui a valu aux journaux qui étaient passés outre, suspensions, poursuites judiciaires et a même donné lieu à emprisonnement de journalistes. La presse en vint, pour contourner ce black out, à se concerter pour publier simultanément des informations que toutes les rédactions avaient confirmées, le gouvernement ne 
pouvant réprimer les "contrevenants". La presse internationale a aussi beaucoup subi. Malgré les velléités d'ouverture, elle continue d'endurer l'obstruction des autorités d'Alger qui se manifestent à travers la procédure de délivrance des visas et les conditions d'exercice de la profession de journaliste. Même en matière de communication, la politique du pouvoir est désastreuse : cela va de la falsification des faits (bilans toujours très en deçà de la réalité) aux réactions tardives par rapport à l'événement, voire à l'absence de réaction officielle même quand le nombre de victimes est important. Puis s'est installée une démarche ambivalente où alternent silence profond des médias sous tutelle et avalanches subites d'images horribles. Cette instrumentation de l'information sécuritaire obéit aux intérêts du moment et aux dividendes que le pouvoir peut compter engranger dans l'opinion. Il ignore totalement le droit des Algériens à l'information et ne se sent nullement lié par l'obligation d'informer. Le comble du black out aura été atteint pendant le mois de ramadan de l'année 2000, tandis que les massacres à grande échelle reprenaient de plus belle. Pour le pouvoir, il n'était pas question de ternir la prétendue réussite de la politique de "concorde civile" chère au Président Bouteflika. Décidée par Bouteflika en septembre 1999, cette mesure a été un appel aux groupes islamistes armés à déposer les armes en contrepartie d'une clémence et d'une réinsertion dans la vie civile. Ceci permit, selon des chiffres communiqués par le pouvoir, à plus de 4000 terroristes de descendre des maquis et aux chefs de blanchir leur "butin de guerre" dans des activités commerciales et immobilières. La plupart ne fut pas inquiétée par la justice, au désespoir des familles des victimes des groupes armés. C'est ainsi que dans de nombreuses localités, bourreaux et familles de victimes se côtoyèrent.

La longue transition politique et le processus de "parachèvement des institutions", selon la formule utilisée par le pouvoir, ont été une occasion pour certains milieux d'affaires, profitant du climat de violence et du terrorisme, de se lancer dans la création de sociétés de sécurité et de gardiennage auprès des entreprises. Depuis 1992, des milliers d'entreprises du secteur privé ont été rackettées par des groupes armés et des centaines d'autres, du secteur public, ont été détruites par des incendies criminels. Elles n'étaient pas organisées pour faire face à pareille menace. Elles ont donc été obligées de consacrer de gros budgets à la protection de leurs employés et de leurs installations, soit en se dotant de leurs propres services de sécurité, soit en faisant appel à des services extérieurs, ce qui fut le plus souvent le cas. Nous retrouvons là des hauts dignitaires de l'armée et des hauts fonctionnaires de police, à la retraite ou en activité. Ils ont imposé leur offre de service aux entreprises les plus sensibles et même à celles qui se situaient dans des zones industrielles déjà protégées. Le marché a été pris d'assaut et quadrillé. Les candidats potentiels ont usé de leurs relations personnelles, parentales et professionnelles, dans les secteurs économiques visés. Ainsi de nouvelles chasses gardées se sont-elles créées, entretenues par des pratiques opaques. Un autre secteur, lui aussi en relation avec la violence, s'est développé considérablement en l'espace de quelques années : l'importation d'équipements de sécurité à destination du secteur civil. Des milliers de systèmes de contrôle (comme des scanners) ont été commercialisés par des sociétés privées sous la coupe des responsables des services de sécurité. Ces sociétés ont acquis une situation de monopole et ont pu imposer leur prix sur un matériel parfois désuet. Qui homologue, agrée et vérifie la qualité de l'équipement? Comment les administrations, les institutions publiques et les entreprises se sont-elles approvisionnées en produits aussi sensibles? A notre connaissance, elles n'ont jamais lancé d'avis d'appel d'offres, comme les y oblige le code 
des marchés publics. Même la pratique du gré à gré, conformément à la loi, suppose des contacts avec plusieurs fournisseurs. Là encore l'alibi sécuritaire et l'urgence de disposer de moyens de répression ont été utilisés et mobilisés à souhait.

La facture à l'importation d'armes et d'équipements de sécurité est estimée à 3 milliards de dollars chaque année depuis l'apparition du terrorisme et de la violence (les chiffres varient selon les sources). Les montants officiels ne sont pas publiés, mais cette somme est supérieure à la facture alimentaire à l'importation, qui se situe entre 2 et 2,5 milliards de dollars. Il est de notoriété que dans le secteur de l'armement, la grande corruption se pratique à des niveaux et des montants très élevés. L'Algérie n'y échappe pas, qui plus est pour avoir été longtemps sous embargo international pendant les années 90. Ainsi, au plus fort de la violence et du terrorisme, faisait-elle son marché d'armements dans des circuits parallèles en passant par une kyrielle d'intermédiaires.

Émeutes populaires et luttes radicales pour la démocratie et les droits de l'homme Depuis quelques années, la société civile mène de multiples combats pour une vie meilleure. Elle exige des administrations locales voire centrales, de rendre compte de leurs activités et de l'utilisation des énormes ressources financières. La revendication pour le droit à plus de progrès et de justice sociale s'est souvent exprimée dans des émeutes de plus en plus fréquentes.

15 A croire que c'est devenu le seul moyen de se faire entendre d'un pouvoir de plus en plus sourd et de plus en plus coupé des réalités. Pour Abdou Benziane, chroniqueur dans plusieurs quotidiens algériens, "actuellement, l'Etat et la société vivent un divorce prolongé, l'un instrumentalisé pour réprimer l'autre sinon l'asservir" (2004). Analysant le sens à donner à la multiplication des émeutes populaires contre le pouvoir, il considère que "l'émeutier parle de sa mal vie sur terre, de son chômage, de sa précarité. Lui opposer le troisième âge, l'arrogance, le policier ou l'allégeance, c'est prévoir pour le pays de nouvelles impasses".

16 La mouvance dite démocrate et républicaine (souvent en rangs dispersés), essaye de maintenir sa présence dans l'espace politique, de plus en plus concurrencée par les nouvelles générations citoyennes, à l'image du mouvement des "aarouch". Ce mouvement, dont la dénomination renvoie aux formes traditionnelles d'organisation de la société en tribus et en comités des sages, est principalement Kabyle. Ces revendications, fortement culturelles et identitaires mettent en avant des aspirations à une meilleure redistribution des richesses nationales, n'hésitant pas à radicaliser ses méthodes de lutte dont l'affrontement direct avec les forces de police et de gendarmerie n'est pas des moindres. Depuis 2001, les "aarouch" sont entrés en conflit direct avec le pouvoir, ce dernier ayant réprimé sauvagement les multiples manifestations populaires en faisant plus de 120 morts. Pour les "aarouch", il s'agissait de mettre un terme à la corruption qui sévit au sein des institutions de l'Etat. Le 14 juin 2001, une imposante manifestation ayant regroupé plusieurs centaines de milliers de personnes à Alger fut durement réprimée par la police (selon un témoignage sur une chaîne de télévision française le 19 juin 2001, à l'occasion d'une émission spéciale sur l'Algérie). Parmi les slogans écrits et clamés lors de cette démonstration de force, on pouvait relever : "le pouvoir assassin" et "Vous ne pouvez pas nous tuer, nous sommes déjà morts". L'année 2001 aura été fortement marquée par des émeutes, un peu partout en Algérie, avec chaque fois le même scénario: affrontements avec les forces de sécurité, répression, emprisonnements, torture, selon de nombreux témoignages, procès expéditifs et lourdes condamnations. Ces dernières années, le pouvoir n'a pas 
lésiné pour se donner les moyens de réprimer en tout lieu et à tout moment. Recrutements massifs, acquisition d'équipements lourds, multiplication à l'intérieur du pays de casernes de forces anti-émeutes à proximité des principales villes ont été les maîtres mots d'une politique répressive financée par d'excellentes recettes pétrolières.

Même dans les régions d'exploitation pétrolière du sud, souvent appelées "l'Algérie utile", pourtant sous haute surveillance, des émeutes pour l'emploi, l'accès au logement social, l'eau et une plus juste répartition des richesses, se multiplièrent. Le pouvoir répondit d'abord et souvent exclusivement par l'usage de la force et l'emprisonnement des leaders supposés. La répression se fît aussi contre la presse et les journalistes qui se firent l'écho de ces évènements. A l'image de ce correspondant de presse et militant actif des droits de l'homme, qui lors du printemps 2004, se retrouva en prison avec plus d'une vingtaine de procès en cours, pour avoir publié des enquêtes sur des malversations impliquant des élus locaux et des représentants de l'Etat! Ou de ces jeunes émeutiers originaires d'un village des Aurès, qui, ayant suspecté leur maire et ses adjoints d'avoir détourné un financement de l'Union Européenne destiné au développement de leur commune, manifestèrent violemment en juin 2004 et se retrouvèrent, manu militari, en prison.

La presse privée, cible des groupes terroristes et du pouvoir

Bien qu'ayant été réprimées avec violence, les manifestations populaires d'octobre 1988, en imposant l'ouverture pluraliste, ont permis à la presse privée de jouer un rôle important dans la révélation des affaires de corruption depuis 1990. Les soixante journalistes assassinés en Algérie entre 1993 et 1997 ne travaillaient pas tous sur des dossiers de corruption.

Leur élimination visait à briser l'essor de la jeune presse, à instaurer un climat de terreur au sein des rédactions, à les vider de leurs journalistes et à instaurer une psychose parmi les rédacteurs qui osaient dénoncer la corruption. L'entreprise a atteint son but: hormis quelques téméraires traités de suicidaires par leurs pairs, les journalistes ont longtemps évité de parler de tout ce qui s'apparente à un détournement de deniers publics ou à la prévarication.

20 Les journalistes d'investigation subissent aujourd'hui, comme tous leurs confrères, la répression des autorités et sont confrontés aux même risques face à la violence, mais s'exposent en plus à ceux que l'on nomme "les barons de la mafia". Ce fut le cas pour Abdelhaï Beliardouh, correspondant local du quotidien El Watan, qui fut kidnappé le 20 juillet 2002 à Tébessa par un groupe d'hommes armés, roué de coups et torturé. Ce jour-là, El Watan publia un article dans lequel le journaliste faisait allusion à l'arrestation du président de la chambre de commerce et d'industrie locale "supposé avoir des liens avec des réseaux terroristes". L'importateur fut immédiatement soupçonné d'avoir été à la tête du groupe d'assaillants. L'incident déchaîna l'opinion publique et entraîna les condamnations d'organisations de droits de l'homme, de partis politiques et de médias algériens et étrangers. Malgré l'action en justice intentée par Béliardouh, toujours en cours et constamment différée, le meneur présumé du groupe a été libéré. La seule mesure prise par les autorités fut de démettre l'importateur de ses fonctions de président de la chambre de commerce et d'industrie. Traumatisé et découragé, Abdelhaï Béliardouh mit fin à ses jours en avalant de l'acide après un mois d'agonie. Transparency International (pour la lutte contre la corruption) lui a décerné à titre posthume en 2003, son "Prix d'Intégrité". 
endant, les difficultés du travail d'investigation et le souci de ne pas fournir de prétexte aux menaces émanant du pouvoir servent aussi parfois d'alibis pour ne pas écrire sur des sujets qui fâchent. Mener des enquêtes de presse est une tâche particulièrement ardue en Algérie en raison du contexte de violence et d'insécurité permanentes. Et, ce ne sont pas seulement les journalistes de la presse privée qui sont inquiétés lorsqu'ils font leur travail. C'est le cas notamment de ce correspondant de l'agence officielle de presse, APS (Algérie Presse Service), dans l'extrême sud du pays, qui fut d'abord tracassé puis emprisonné suite à une dépêche "hors service", c'est-à-dire une information qui n'était pas destinée à sortir de l'agence.

Où en est aujourd'hui la presse algérienne de statut privé? Les assassinats de journalistes ont heureusement cessé et les menaces physiques sur les professionnels de la presse se font de moins en moins fréquents. Néanmoins, les conditions d'exercice de leur métier demeurent extrêmement difficiles pour beaucoup d'entre eux. Le vendredi 20 février 2004, tous les "imams" des mosquées contrôlées par le gouvernement, sur instruction du pouvoir, se sont donnés le mot en prenant la presse indépendance pour cible : un véritable appel au crime et une chasse aux sorcières sont lancés du haut des minarets. Un discours incendiaire est même transmis en direct à la télévision à partir de la mosquée de Constantine.

Le dessinateur du quotidien Liberté, Ali Dilem, déjà poursuivi en justice par le ministère de la défense nationale pour une vingtaine de plaintes depuis trois ans, est nommément visé dans les prêches des islamistes. Le ministre algérien du culte a confirmé que ces discours répondaient aux orientations données par son département. C'est dans ce climat d'inquisition des plus explosifs, que le président Bouteflika annonçait en février 2004 sa candidature à un second mandat présidentiel. Pour être reconduit, il était prêt à tout, quitte à pactiser avec les intégristes islamistes. Bouteflika, qui n'a jamais caché son hostilité à la liberté d'expression, a garanti son succès grâce à une forte fraude annoncée et un taux d'abstention record. Il faut noter cependant que le chef du gouvernement algérien a vite désavoué son ministre du culte lors d'une conférence de presse. Des journalistes sont de nouveau emprisonnés en 2004, parmi lesquels Mohamed Benchicou, directeur du quotidien Le Matin et auteur d'un ouvrage intitulé Bouteflika, une imposture algérienne (1994). L'impression de son quotidien est alors interdite.

Le culte du secret et l'injustice au pouvoir

Côté pouvoir - tous clans et officines confondus - le secret est devenu une sorte de seconde nature. L'historien Benjamin Stora évoque ainsi "les imaginaires du secret" à l'œuvre dans la situation algérienne actuelle (1998). S'interrogeant sur les forces en conflit, il se demande "quels sont les acteurs réels du pouvoir qui font de l'opacité une arme essentielle de gouvernement" et considère qu'en Algérie le secret est pensé comme "secret de fabrication" de la politique, incompatible avec le principe de la "chose publique". Il s'agit d'un système où un groupe d'hommes se dissimule derrière un épais rideau pour contrôler le pays (Stora 1998).

Prenons l'exemple de la lutte contre la corruption: elle est avant tout politique. Information, transparence, contrôle, réforme, participation populaire, citoyenneté sont les maîtres mots d'une avancée nécessaire qui se déclinerait en libertés à conquérir, en responsabilités à prendre, en ouvertures du pouvoir à d'autres secteurs de la société. Il faudrait pratiquer des brèches dans le mur bétonné du silence, redéfinir la loi pour ramener les institutions près du peuple, casser les monopoles politiques, militaires et

Bulletin de I'APAD, 25 | 2003 
économiques pour donner à cette société la possibilité de se battre pour elle-même et de devenir une société de citoyens et enfin, déstructurer les réseaux de la corruption, mais la justice est complètement inféodée au pouvoir. Elle a perdu toute crédibilité aux yeux de l'opinion publique. Miloud Brahimi (1998), avocat et fondateur de la première ligue algérienne des droits de l'homme, considère "que le fonctionnement de l'appareil judiciaire est inséparable de celui d'un système qui ne manifeste aucune hâte à se débarrasser des réflexes du passé, ni d'un héritage textuel dont il fait l'usage que l'on sait". Il constate aussi, dans le même article, que "l'indépendance de la justice, entrevue entre 1989 et 1992, n'est pas à l'ordre du jour. Il en sera ainsi tant que le processus de démocratisation ne connaîtra pas une avancée significative". L'état des lieux en 2004 confirme que non seulement il n'y a pas eu de changement, que les faits confirment l'analyse de cet avocat, mais qu'au contraire, la situation s'est même aggravée depuis. La justice ne continue-t-elle pas à être utilisée pour tenter de caporaliser la liberté d'expression, réduire à néant le pluralisme syndical (interdiction des grèves), maîtriser la fronde interne des magistrats (en réprimant leur syndicat légitime), porter le coup de grâce au secteur public économique (en emprisonnant ses dirigeants), violer le droit des citoyens, piloter une campagne officielle anti-corruption qui épargnait les véritables milieux mafieux et piétiner les droits de l'homme?

Les prédateurs s'attaquent à la côte algérienne "aidés" par les massacres de populations

En marge du forum économique de Crans-Montana en Suisse, édition de l'automne 1999, le président Bouteflika affirmait, lors d'une conférence de presse, que "le marché touristique algérien est une affaire de plusieurs milliards de dollars et que l'Algérie entend engager des pourparlers avec des partenaires étrangers désirant collaborer sérieusement et investir dans ce secteur vital de notre économie". Certains milieux d'affaires à l'affût de toutes les opportunités juteuses n'ont pas attendu cet appel pour s'accaparer le créneau. Sur la côte algérienne, l'OPA au dinar symbolique a commencé depuis plusieurs années, au plus fort de la violence et des tueries menées par les islamistes armés. Les mille deux cents kilomètres de littoral algérien sont la seule partie du sud-ouest de la Méditerranée encore relativement épargnée par le béton. Pas pour longtemps, car le pillage a commencé. La stratégie employée par ces investisseurs est toute simple : lancer des projets touristiques fumeux pour s'approprier les sites, tout en bénéficiant des facilités accordées par la loi et les organismes d'aide à l'investissement. C'est le moyen de devenir propriétaire des lieux et intermédiaires des investisseurs étrangers qui pointent le nez, venant des pays du Moyen Orient et du Golfe plus particulièrement. L'un d'entre eux, issu de la famille Hariri du Liban et proche du Premier ministre, n'a pas caché son intérêt, lors d'une visite en Algérie, à investir dans des projets touristiques et commerciaux en partenariat avec des entreprises publiques algériennes ${ }^{1}$. L'échec du projet lui fit dire : "je crois d'ailleurs qu'on a utilisé le nom de Rafik Hariri [nom du Premier ministre libanais] pour avoir une OPA sur ces terres et lever tous ces obstacles. Cette zone [la côte] est sans doute au centre de nombreux enjeux politiques". Ce qui est acquis pour une bouchée de pain, par les voies les plus "légales" au demeurant, mais avec des complicités certaines au sein des institutions concernées de l'Etat, sera revendu au prix fort aux investisseurs venus d'ailleurs, annonçant une spéculation foncière effrénée sur tout le littoral. A moins que l'acquéreur opportuniste ne se pose en associé des partenaires étrangers professionnels. départs massifs des populations de ces régions côtières, qui ont été particulièrement 
ciblées par des tueries et des massacres de civils. Entre 1994 et 2000, les habitants se sont vus contraints à s'installer dans des conditions très précaires à la périphérie des grandes villes. Ce dépeuplement a facilité l'accaparement des zones touristiques potentielles. Un climat de psychose et de terreur, souvent mis à l'actif des groupes islamistes armés, s'en suivit pendant plusieurs années. Même les bergers n'ont pas échappé à cette violence. Des chefs de milices locales dites antiterroristes et armées par les militaires se permirent même d'accaparer des terres du domaine maritime pourtant inaliénables au regard de la loi. La localité de Béjaïa, à trois cents kilomètres à l'est d'Alger, fut dénaturée par des constructions bétonnées abritant des lieux de beuverie et de débauche. Ceci était, selon un entretien, le prix à payer "pour les sacrifices consentis". Cet exemple n'est malheureusement pas unique. Dans le même temps, sécuriser ces régions et protéger les populations locales étaient loin d'être des priorités, les rescapés n'avaient qu'un seul choix : prendre le chemin de l'exil vers des cieux plus cléments.

Les affaires continuent pendant la guerre civile

Pourquoi la persistance des massacres contre les populations ? La facilité déconcertante avec laquelle les groupes islamiques armés ont opéré de longues années durant, a permis aux islamistes politiques de remettre sur le tapis la question de "qui tue qui", accusant implicitement le pouvoir et ses multiples services de sécurité d'être derrière ces tueries. Ou autre question: existe-t-il une stratégie visant à faire perdurer le terrorisme ? Cette stratégie pourrait être le fait des cercles du pouvoir qui ont intérêt à ce que le cycle violence-répression soit régulièrement entretenu. Qu'on ne dise pas que la chose est impensable: elle est avérée en ce qui concerne l'Italie des années soixante-dix. Ce bel exemple de symbiose entre pouvoir corrompu et cercles terroristes au sein du pouvoir est analysé par Ferdinando Imposito, ancien magistrat italien et avocat pénaliste spécialisé dans les affaires de corruption et de violations des droits de l' homme. Dans la préface de La sale guerre (2001), il écrit à propos de l'Algérie que "l'histoire de ces dernières années (...) est riche en épisodes révélant cette imbrication de canaux souterrains qui finissent toujours par trouver une voie de communication entre eux". Ce juriste a découvert à la lecture du livre "que nombre des protagonistes de La sale guerre, ceux-là mêmes qui ont eu les plus grandes responsabilités dans la stratégie de la tension, dans un camp comme dans l'autre, se sont enrichis et sont devenus des oligarques intouchables".

Il faut rappeler que la mouvance islamiste en Algérie n'est pas une création ex nihilo des années quatre-vingt. Elle s'inscrit dans une tradition déjà longue, dont les sources récentes datent de l'indépendance. Ce sont des organisations, un éventail de tendances, de luttes intestines, un écheveau de fils et de liens multiples avec les hommes du pouvoir, ne serait-ce que parce que certains des plus anciens activistes de l'islamisme firent partie du FLN. La réalité entre islamisme et corruption est complexe. Le leader de Hamas, mouvance fondamentaliste, déclarait en 1998 "la nécessité de lutter contre la corruption", que le pouvoir ne "faisait pas grand chose pour lutter contre ce fléau". Il ajoutait que "les plus de 30 milliards de dollars de dette de l'Algérie doivent être assimilés à un vol organisé des richesses de l'Algérie".

Luis Martinez (1998) fait état d'une instrumentalisation de l'alternative islamiste par la jeunesse algérienne qui y adhère. En effet, l'islamisme "laissant le champ économique aux initiatives individuelles", correspond bien aux "désirs de richesse" de cette jeunesse laissée pour compte par les gouvernements algériens. 
que cet "enrichissez-vous" s'est transcrit dans certaines mouvances islamistes par un "enrichissez-nous" débouchant sur tous les trafics et les rackets. Louis Martinez confirme ce glissement : le rôle de protection de la population qu'ont prétendu jouer les GIA (Groupes islamiques armés) dans les communes qui leur étaient acquises s'est mué en une forme de prédation, "la pratique du racket se généralisant entre 1993 et 1994". Puis la destruction d'entreprises économiques par les groupes armés, à l'exemple des cimenteries, a favorisé les réseaux d'importation aux mains de la mafia. Pour Luis Martinez, la pérennité de la violence s'explique par la "mutation vers le trafic de certains groupes islamistes (...), car elle permet l'accroissement de leur pouvoir d'achat, notamment en devises, et donc la disposition de matériels de guerre plus sophistiqués".

Boutamine, avocat algérien, affirmait dans un entretien à la presse qu'en Algérie, "situation assez particulière, certains milieux de la corruption et leurs pratiques mafieuses ont fait, depuis quelques années, jonction avec les agissements des organisations intégristes légalisées, même celles qui étaient clandestines au départ. Aujourd'hui, le terrorisme et même la subversion islamiste couvrent et protègent le développement de la corruption et de la contrebande ${ }^{2 "}$. Il rappelait les conclusions de la réunion internationale sur ]e terrorisme qui ont "apporté la preuve de la collusion entre les milieux de la corruption et du trafic de drogue et les réseaux subversifs islamistes. Cette subversion a été pour beaucoup dans le développement du trafic et de la corruption : gros trahendo (contrebande), la fraude fiscale, la fuite des capitaux...".

Le "qui tue qui" se redouble d'un "qui finance qui". Et cela ne fait qu'ajouter aux doutes et aux perplexités. La certitude qui nous reste est celle de l'incapacité, tant du pouvoir actuel que des islamistes à mettre fin au monstrueux état de choses que connaît l'Algérie. C'est ce que confirment les propos de Lahouari Addi qui, se référant à la démarche du pouvoir qui a fait du pétrole un instrument de dépendance, note: "la nature rentière et distributive de l'économie est un obstacle autrement plus important que l'islam à la participation politique" (1998). Les chefs des partis islamistes se sont lancés dans les affaires, à l'image de l'ancien leader de Hamas, feu Mahfoud Nahnah, qui aspirait de plus en plus à jouer dans la cour des grands de "l'import-import", à s'acoquiner avec des généraux, impliqués eux-mêmes ou à travers leur progéniture, et à tirer de gros profits de toutes sortes de trafic. La "concorde civile" a été l'occasion, dès le lendemain du référendum de septembre 1999, pour les chefs repentis de groupes islamistes armés de blanchir leur butin de guerre, notamment dans le commerce et l'immobilier, affichant sans vergogne un train de vie ostentatoire et narguant les familles des victimes du terrorisme.

Ainsi, pouvoir militaire et islamisme se retrouvent-ils sur le fond : leur conception de la politique est la même, totalitaire et mafieuse ; leurs différends portent essentiellement sur le partage du pouvoir et des prébendes qui lui sont attachées. Les hommes du pouvoir à tous les niveaux ne cessent de se servir, à l'image de ceux qui ont été placés à la tête des municipalités, en remplacement des maires islamistes suite à la dissolution des conseils municipaux en septembre 1992. Les tares de ce type de gestion (système qui perdura jusqu'en 1997) sont rapidement apparues. La plupart de ces fonctionnaires désignés et leurs acolytes se transformèrent en corrompus, dirigeant leur mairie comme bon leur semblait, dilapidant les budgets et lançant des opérations coûteuses en vue des dividendes qu'ils retireraient. La presse publia de nombreuses enquêtes à ce propos et la justice fut saisie dans quelques cas seulement. La totalité de ces maires

Bulletin de I'APAD, 25 | 2003 
désignés par le pouvoir étant armés et disposant même parfois de gardes du corps, voire de milices, il leur arrivait, sous couvert de traquer les terroristes et leurs réseaux de soutien, de lancer des actions punitives contre des citoyens sans défense et des personnes qui auraient pu dénoncer leur gestion catastrophique. Le pouvoir finit par reconnaître l'existence de ces dérives et ces dépassements, suite aux révélations de la presse et des dénonciations des victimes.

Puis il y eut d'inimaginables tractations : des postes de maires ont été "achetés" par des gros commerçants de l'import-import", qui faisaient ainsi un "investissement" à rentabiliser rapidement, plus particulièrement dans les communes du littoral, à la périphérie des grandes villes. Beaucoup de ces "OPA" eurent lieu entre 1994 et 1996. Des intermédiaires, déjà associés dans des affaires illégales, agissaient auprès des hauts fonctionnaires de l'Intérieur et des collectivités locales. Les barons du système géraient directement la nomination et l'affectation de ces maires non élus, se partageant les communes les plus juteuses et élargissant leur zone d'influence. Aux élections municipales d'octobre 1997, malgré la fin de la transition et grâce à une fraude massive (reconnue même par une commission d'enquête parlementaire), beaucoup de ces maires furent reconduits par les urnes pour cinq ans. L'armée - le pouvoir réel - se donnait ainsi une légitimité démocratique.

Les années 2000 : criminalités et explosion des recettes pétrolières Si le début du XXI ${ }^{\mathrm{e}}$ siècle voit se confirmer pour l'Algérie l'embellie pétrolière réapparue dès 1999, la même période est marquée par une très nette recrudescence des affaires de grande corruption, notamment dans les transactions commerciales internationales, ce que confirment des enquêtes publiées par la presse et plusieurs études spécialisées, menées pour le compte de Transparency International notamment. Nombre d'observateurs font le lien de proportionnalité entre les recettes issues du pétrole et la multiplication de ces scandales. Si le pouvoir algérien est très peu disert sur ces questions, il reconnaît quand même l'existence et l'essor du crime organisé sous toutes ses facettes: de la contrebande à très grande échelle (la cigarette notamment dans le sud du pays, en liaison avec la région du Sahel), du trafic de drogue particulièrement florissant, du développement de la délinquance économique et financière, de l'apparition du grand banditisme, du grand nombre d'armes en circulation, etc. Ce que l'on a appelé l'affaire de la banque privée Khalifa - énorme opération de blanchiment d'argent et de fuite de capitaux -, a mis à nu à la fois la déliquescence qui frappe les institutions de contrôle en Algérie et les complicités qui existent au sein de l'Etat. Les conséquences dramatiques en vies humaines - plus de trois milles victimes et des dizaines de milliers de sans-abri dues au séisme qui a touché la région d'Alger en mai 2003 et l'effondrement de centaines d'immeubles, ont démontré l'ampleur de la corruption qui gangrène le secteur de 1 'habitat, de la construction et de la commande publique. Que font les pouvoirs publics pour mettre un terme à toutes ces pratiques criminelles? Sous la pression internationale et de ses principaux bailleurs, le pouvoir algérien se contente de signer des conventions contre la corruption, ne manifestant aucune volonté politique d'aller plus loin pour traduire ces conventions en mesures concrètes dans la vie du pays. Visiblement, les pays qui sont ses principaux clients et ses plus importants fournisseurs ne s'empressent pas non plus d'exiger plus de transparence dans leurs échanges commerciaux.

Conclusion 

évènements qui secouent l'Algérie depuis 1992? Excellant dans l'art de la manipulation, jouant du reversement de ses alliances, tantôt avec le courant démocratique, tantôt avec les islamistes, il a régulièrement préféré coller à la conjoncture. Pour ce faire, il s'est habillé de conservatisme, utilisant ce qu'il appelle "les constantes nationales" (l'unité nationale, la langue arabe, l'islam, et les acquis du 1er novembre 1954, date de déclenchement par le FLN de la guerre d'indépendance) comme un fonds de commerce dans lequel il puise pour ramener dans son giron les courants islamistes - qui ne sont ni plus ni moins mieux musulmans que le commun des Algériens pratiquants. La mission des islamistes politiques est d'agir pacifiquement, aussi bien en intégrant le gouvernement qu'en se situant dans l'opposition parlementaire. Les uns, beaucoup plus rentiers que conservateurs, les autres islamistes conjoncturels, captent le rejet du pouvoir et les frustrations des Algériens marginalisés. Mais alors où est la solution au drame algérien? Fernando Imposimato (2001) considère que la résolution de cette crise sera longue et difficile mais il est pessimiste quant aux chances de succès de la démarche pour y parvenir, telle que préconisée par le pouvoir en Algérie: "Face aux massacres de la population civile qui perdurent dans une situation économique et sociale détériorée et dramatique marquée par la corruption et la délinquance organisée, il n'est pas pensable que la politique dite de concorde civile du président Bouteflika, ne prenant pas en compte les problèmes politiques, puisse mettre fin au bain de sang".

Pour Daniel Sibony ${ }^{3}$, psychanalyste, à propos des questionnements sur l'origine des violences actuelles en Algérie, le retour à l'histoire est nécessaire. Il considère d'abord que les deux analyses courantes - le chaos est dû aux islamistes/l'Etat algérien est tout aussi fanatique - "sont fausses parce que rationnelles". L'hypothèse qu'il émet se réfère à l'Algérie qui "se meurtrit pour changer de peau et se dégager de sa plénitude ; pour rendre enfin possibles des luttes qui ne soient pas des meurtres; pour permettre d'autres violences que narcissiques". Benjamin Stora ${ }^{4}$ est plus circonspect: il doute "d'un possible Etat de droit, émergeant des massacres et des ruines de l'esprit". Pour Luis Martinez (1998), "si la guerre civile représente pour les en-bas une occasion d'ascension sociale, pour les en-haut, elle constitue une période de recomposition des privilèges et d'accroissement de la richesse". Quel dépassement de la violence chaotique est-il en effet possible aujourd'hui en Algérie? La situation algérienne peut paraitre désespérée, mais ce serait oublier que ce pays a, au cours de son histoire, résolu des problèmes bien plus lourds, par exemple pour sortir des brèches coloniales.

De son côté, le peuple résiste de mille manières à ceux qui dirigent le pays et le mènent au chaos. La peur recule chez des millions d'Algériens, malgré la persistance de toutes sortes de violences et d'atteintes aux droits de l'homme : la lutte pour la survie et pour la liberté se poursuivent. Les conditions d'un sursaut pour arrêter le pillage et faire cesser toutes les violences sont-elles réunies? La société dans son ensemble a-t-elle suffisamment conscience de l'ampleur de la corruption qui s'apparente à une mise à sac du pays, hypothéquant la perspective de développement ? L'avenir nous le dira. 


\section{BIBLIOGRAPHIE}

Addi L., 1998, "L'islam est-il soluble dans la démocratie ?", Paris, La Découverte, Mouvements, nº 1.

Benchicou M., 1994, Bouteflika, une imposture algérienne, Alger, éditions Le matin, Benziane A., 2004, "La légitimation de l'émeute", Le quotidien d'Oran, 11 mars 2004.

Brahimi M., 1998, "La justice, entre archaïsme et modernité", Les Cahiers de l'Orient, numéro spécial "Algérie", Paris.

Gèze F., 2002, "Françalgérie : sang, intox et corruption", Mouvements n²1-22, Paris.

Hadjadj D., 2001, Corruption et démocratie en Algérie, Paris, ed. La dispute.

Imposimato F., 2001, "Préface", in : Souaïdia H., La sale guerre, Paris, La découverte.

Martinez L., 1998, La guerre civile en Algérie, Paris, Karthala.

Péan P., 1988, L'argent noir. Corruption et sous-développement, Paris, Fayard. Souaïdia H., 2001, La sale guerre, Paris, La découverte.

Stora B., 1998, Algérie, naissance d'une nation, Paris, éditions Atlantica.

\section{NOTES}

1.Le Matin, 8 avril 2003.

2.Témoignage de Maître Boutamine dans Le matin, 15 octobre 1998.

3.Sibony D., "Algérie : une étrange violence". Libération. 6 avril 1998.

4.Benjamin Stora,"Retour à Constantine. entre histoire et mémoire", Libération. 13 mai 1998.

\section{AUTEUR}

\section{DJILALI HADJADJ}

Journaliste et auteur de Corruption et démocratie en Algérie, Paris, La Dispute, 2001. 